\title{
A Taxonomy of Similarity Mechanisms for Case-Based Reasoning*
}

\author{
Pádraig Cunningham \\ University College Dublin
}

\author{
Technical Report UCD-CSI-2008-01 \\ January $6^{\text {th }} 2008$
}

\begin{abstract}
Assessing the similarity between cases is a key aspect of the retrieval phase in casebased reasoning (CBR). In most CBR work, similarity is assessed based on feature-value descriptions of cases using similarity metrics which use these feature values. In fact it might be said that this notion of a feature-value representation is a defining part of the CBR world-view - it underpins the idea of a problem space with cases located relative to each other in this space. Recently a variety of similarity mechanisms have emerged that are not founded on this feature-space idea. Some of these new similarity mechanisms have emerged in CBR research and some have arisen in other areas of data analysis. In fact research on Support Vector Machines (SVM) is a rich source of novel similarity representations because of the emphasis on encoding domain knowledge in the kernel function of the SVM. In this paper we present a taxonomy that organises these new similarity mechanisms and more established similarity mechanisms in a coherent framework.
\end{abstract}

\section{Introduction}

Similarity is central to CBR because case retrieval depends on it. The standard methodology in $\mathrm{CBR}$ is to represent a case as a feature vector and then to assess similarity based on this feature vector representation (see Figure 6(a)). This methodology shapes the CBR paradigm; it means that problem spaces are visualised as vector spaces, it informs the notion of a decision surface and how we conceptualise noisy cases, and it motivates feature extraction and dimension reduction which are key processes in CBR systems development. It allows knowledge to be brought to bear on the case-retrieval process through the selection of appropriate features and the design of insightful similarity measures.

However, similarity based on a feature vector representation of cases is only one of a number of strategies for capturing similarity. From the earliest days of CBR research more complex case representations requiring more sophisticated similarity mechanisms have been investigated. For instance, cases with internal structure require a similarity mechanism that considers structure $[1,2]$. More recently a number of novel mechanisms have emerged that introduce interesting alternative perspectives on similarity. The objective of this paper is to review these novel mechanisms and to present a taxonomy of similarity mechanisms that places these new techniques in the context of established CBR techniques.

${ }^{*}$ This research was supported by Science Foundation Ireland Grant No. 05/IN.1/I24 and EU funded Network of Excellence Muscle - Grant No. FP6-507752. 


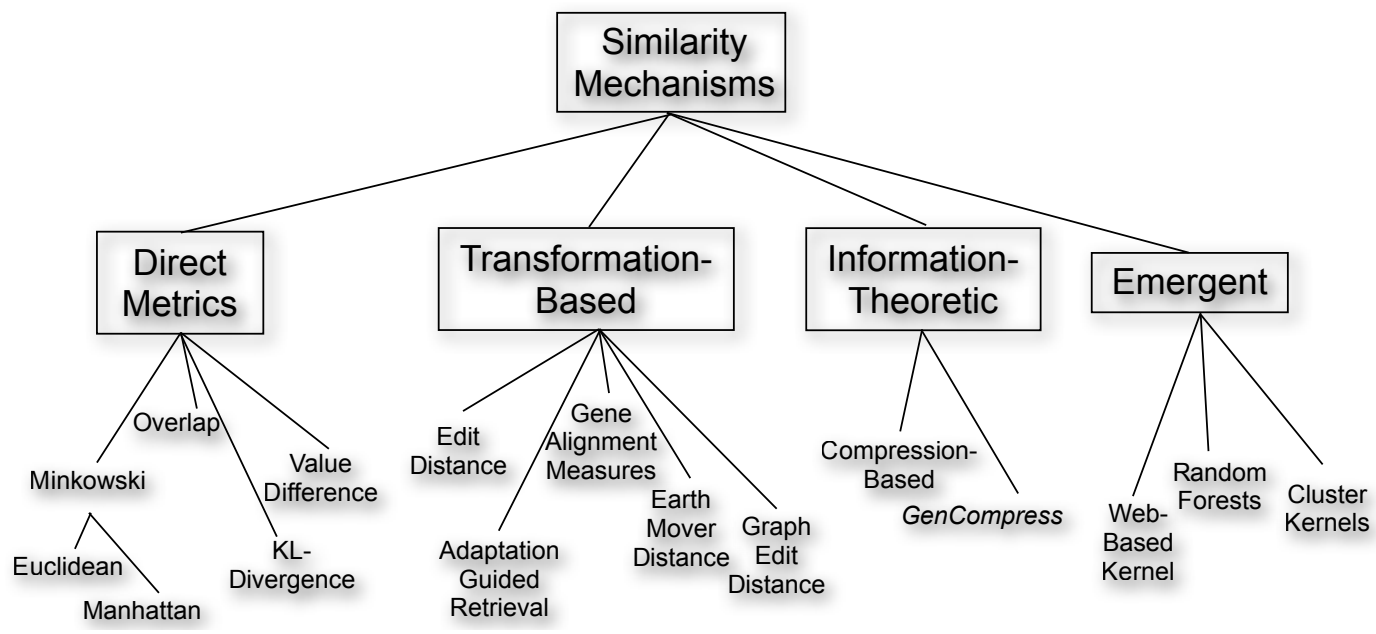

Figure 1: A taxonomy of similarity mechanisms.

Some of these novel similarity strategies have already been presented in CBR research (e.g. compression-based similarity in $[3]$ and edit distance in $[4,5])$ and others come from other areas of machine learning (ML) research. The taxonomy proposed here organises similarity strategies into four categories (see also Figure 1):

- Direct mechanisms,

- Transformation-based mechanisms,

- Information theoretic measures,

- Emergent measures arising from an in-depth analysis of the data.

The first category covers direct mechanisms that can be applied to feature-vector representations and is the dominant well-established strategy. Examples of transformation-based approaches to similarity have been around for some time (e.g. graph edit distance, [1]), however there have been a number of recent developments in this area as the resources for what are normally computationally expensive techniques have come available. The last two categories cover some exciting new directions in similarity research and placing these in the context of other approaches to similarity is the main contribution of this paper.

Strategies for similarity cannot be considered in isolation from the question of representation. For this reason a brief review of representation strategies in CBR is provided in section 2 before we embark on an analysis of similarity in later sections. The review of similarity techniques begins in section 3 where the direct feature-based approach to similarity is discussed. Then transformation-based techniques are described in section 4. Novel strategies based on ideas from information theory are discussed in section 5. In section 6 some novel techniques that we categorize as emergent are reviewed - similarity derived from random forests and cluster kernels are covered in this section. Then in section 7 we review the implications of these new similarity strategies for CBR research. The paper concludes with a summary and suggestions for future research in section 8 . 
Table 1: An example of an internal (or intensional) case representation - case attributes describe aspects that would be considered internal to the case.

\begin{aligned} & \hline Case D \\ & \hline Title: Four Weddings and a Funeral \\ & Year: 1994 \\ & Genre: Comedy, Romance \\ & Director: Mike Newell \\ & Starring: Hugh Grant, Andie MacDowell \\ & Runtime: 116 \\ & Country: UK \\ & Language: English \\ & Certification: USA:R (UK:15) \\ & \hline\end{aligned}

\section{Representation}

For the purpose of the analysis presented in this paper we can categorise case representations as follows:

- Feature-value representations

- Structural representations

- Sequences and strings

In the remainder of this section we summarise each of these categories of representation.

\subsection{Feature-Value Representations}

The most straightforward case representation scenario is to have a case-base $D$ made up of $\left(\mathbf{x}_{i}\right)_{i \in[1,|D|]}$ training samples with these examples are described by a set of features $F$ with numeric features normalised to the range $[0,1]$. In this representation each case $\left(\mathbf{x}_{i}\right)$ is described by a feature vector $\left(\mathbf{x}_{i 1}, \mathbf{x}_{i 2}, \ldots, \mathbf{x}_{i|F|}\right)$.

\subsubsection{Internal versus External}

An important distinction in philosophy concerning concepts is the difference between intensional and extensional descriptions (definitions). An intensional description describes a concept in terms of its attributes (e.g. a bird has wings, feathers, a beak, etc.). An extensional description defines a concept in terms of instances, e.g. examples of countries are Belgium, Italy, Peru, etc. In the context of data mining we can consider a further type of description which we might call an external description. In a movie recomender system an intensional description of a movie might be as described in Table 1 whereas an external 'description' of the movie might be as presented in Table 2. The data available to a collaborative recommender system is of the type shown in Table 2 , it can produce a recommendation for a movie for a user based on how other users have rated that movie. We can think of the data available to the collaborative recommender as an external feature-value description whereas a content-based recommender would use an intensional (or internal) feature-value representation. 
Table 2: An example of a set of cases described by external features. In this example the movie Case $\mathrm{D}$ is described by three external features, the ratings given to it by users 2,3 and 6 .

\begin{tabular}{rrrrrrrr}
\hline & A & B & C & D & E & F & G \\
\hline User 1 & 0.6 & 0.6 & 0.8 & & & 0.8 & 0.5 \\
User 2 & & 0.8 & 0.8 & 0.3 & 0.7 & & \\
User 3 & 0.6 & 0.6 & 0.3 & 0.5 & & 0.7 & 0.5 \\
User 4 & & & & & 0.7 & 0.8 & 0.7 \\
User 5 & 0.6 & 0.6 & 0.8 & & & 0.7 & \\
User 6 & & 0.8 & 0.8 & 0.7 & 0.7 & & \\
User 7 & 0.7 & 0.5 & & & 0.7 & & \\
User 8 & & & & & 0.7 & 0.7 & 0.8 \\
\hline
\end{tabular}

\subsubsection{Representation Enhancement}

A recent trend in CBR, and in ML in general, has been to extend or enhance the case representation derived from the case. Wiratunga et al. have shown how techniques from association rule mining can be used to impute new features for textual cases [6]. They use the a priori association rule learner to discover word associations in a text corpus and then use these associations to add terms to the representation of a text. They show that this enhanced representation improves classification accuracy.

Whereas the work described by Wiratunga et al. extends the representation of individual cases by mining the training corpus, Gabrilovich and Markovitch present a strategy for representation enhancement based on web mining [7]. They employ an auxiliary classifier to associate texts to be classified with articles in Wikipedia. The representation of the texts is augmented with features denoting the concepts associated with these Wikipedia articles. They show that this augmented representation improves the classification performance.

These two approaches to representation enhancement are similar in that they allow texts to be represented by more features. In the first scenario this is done by mining the training corpus to discover new associations, in the second scenario this is done by looking outward to Wikipedia to discover new associations. In either scenario, the end result is still a feature-value representation.

\subsection{Structured Representations}

Practical experience in the development of CBR systems has demonstrated that a simple feature vector is not adequate to represent the complexity of cases encountered in practice: it is often the case that cases have internal structure that needs to be represented $[8,1,9,10,11]$. Given that research on structural case representations is driven by the requirements of practical applications there is considerable variety in the types of structured representation that has been covered in the literature. However the following three categories covers the alternatives fairly well:

- The most straightforward structure (beyond a flat feature vector) is a hierarchical structure whereby the feature values themselves reference non-atomic objects. For instance in case $\mathbf{x}_{i}=\left(\mathbf{x}_{i 1}, \ldots, \mathbf{x}_{i f}, \ldots, \mathbf{x}_{i|F|}\right)$ the feature $\mathbf{x}_{i f}$ could reference a case-structure. This simple extension allows for the description of cases with a complex hierarchical structure $[8,10,12,13]$. 
- The next most general structure is a network structure (typically a semantic network): there is a long history of semantic network-based case representations in CBR and in the related research area of analogical reasoning $[2,14,11]$. Whereas in a hierarchical representation there is only one link type, the part-of link, in a network structure there can be many link types with a rich link semantics.

- The final type of structured representation we distinguish here is the flow structure. These can be cases that represent plans (see Veloso and Carbonell [15]) or workflows as described by Minor et al. [9]. From a structural perspective these flow representations share many of the characteristics of hierarchical and network representations, however since they model an activity they also have a temporal dimension.

These three categories describe situations where cases have an internal structure that is more elaborate than a simple feature vector representation. The other way in which case representations can have structure is when the case vocabulary itself (i.e. the attributes) have a structured representation - for instance if the attribute values are organised into a taxonomy. This scenario is described very well by Bergmann et al $[8,16]$. When the network structure described above is combined with such a rich attribute vocabulary then we have an object oriented case representation $[8,16]$.

\subsection{String and Sequence Representations}

In many circumstances, such as help-desk applications for instance, experiences will be recorded in free text $[17,18]$. An obvious example highlighted by Bergmann is a Frequently Asked Questions list [16]. Such a free text representation greatly influences the strategies for case similarity that can be employed. The most straightforward representation for free text that supports similarity assessment is the bag-of-words strategy from information retrieval.

The bag-of-words approach can be illustrated with the following example. Consider three sentences representing three email messages in a spam filtering scenario:

1. The easiest online school on earth.

2. Here is the information from Computer Science for the next Graduate School Board meeting.

3. Please find attached the agenda for the Graduate School Board meeting.

The first sentence is from a spam message and the other two are from legitimate emails. The bag-of-words representation of these sentences is shown in Table 3. With stop words removed (the, on, here, is, etc.) the sentences are represented as vectors in a 14-dimension space. Thus these strings can be converted to a vector space representation and direct similarity methods can be applied (see section 3). In section 4 we examine transformation-based approaches to string matching and in section 5 we consider information theoretic measures.

\section{Direct Similarity Mechanisms}

The fundamental idea in CBR is problem solving based on the retrieval of similar cases, in its simplest form this is nearest neighbour classification. The intuition underlying this is quite straightforward, examples are classified based on the class of their nearest neighbours. It is often useful to take more than one neighbour into account so the technique is more commonly referred to as $k$-nearest neighbour $(k$-NN) classification where the $k$ nearest neighbours are used 
Table 3: An example of a bag-of-words representation of three simple sentences.

\begin{tabular}{|c|c|c|c|c|c|c|c|c|c|c|c|c|c|c|}
\hline & 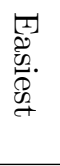 & 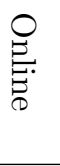 & 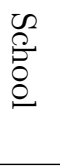 & & $\overrightarrow{\vec{C}^{\circ}}$ & 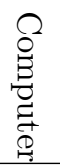 & $\begin{array}{l}\tilde{\Omega} \\
\stackrel{\Omega}{0} . \\
\stackrel{0}{0} \\
\stackrel{0}{0}\end{array}$ & $\begin{array}{l}2 \\
\stackrel{2}{0} \\
\stackrel{0}{0} \\
\stackrel{0}{0}\end{array}$ & 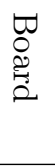 & 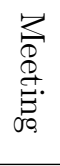 & 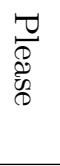 & 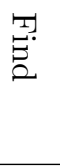 & 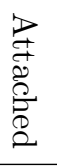 & 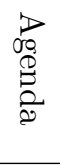 \\
\hline 1 & $x$ & $x$ & $x$ & $x$ & & & & & & & & & & \\
\hline 2 & & & $x$ & & $x$ & $x$ & $x$ & $x$ & $x$ & $x$ & & & & \\
\hline 3 & & & $x$ & & & & & $x$ & $x$ & $x$ & $x$ & $x$ & $x$ & $x$ \\
\hline
\end{tabular}

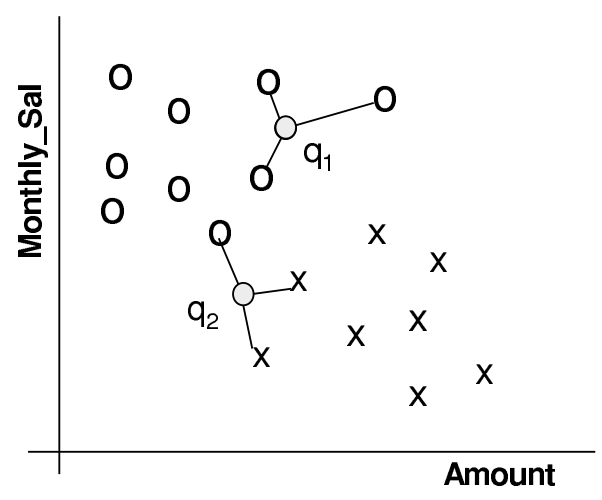

Figure 2: A simple example of 3-nearest neighbour classification.

in determining the class. In most circumstances cases are represented as feature vectors and similarity is assessed directly from these features.

The basic idea is as shown in Figure 2 which depicts a 3 -nearest neighbour classifier on a two-class problem in a two-dimensional feature space. In this example the decision for $q_{1}$ is straightforward - all three of its nearest neighbours are of class $\mathrm{O}$ so it is classified as an $\mathrm{O}$. The situation for $q_{2}$ is slightly complicated at it has two neighbours of class $\mathrm{X}$ and one of class $\mathrm{O}$. This can be resolved by simple majority voting or by distance weighted voting (see below).

So $k-\mathrm{NN}$ classification has two stages; the first is the determination of the nearest neighbours and the second is the determination of the class using those neighbours.

Let us assume that we have a training dataset $D$ made up of $\left(\mathbf{x}_{i}\right)_{i \in[1,|D|]}$ training samples. The examples are described by a set of features $F$ and any numeric features have been normalised to the range $[0,1]$. Each training example is labelled with a class label $y_{j} \in Y$. Our objective is to classify an unknown example $\mathbf{q}$. For each $\mathbf{x}_{i} \in D$ we can calculate the distance between $\mathbf{q}$ and $\mathbf{x}_{i}$ as follows $\left(w_{f}\right.$ is the weight assigned to feaure $\left.f\right)$ :

$$
d\left(\mathbf{q}, \mathbf{x}_{i}\right)=\sum_{f \in F} w_{f} \delta\left(\mathbf{q}_{f}, \mathbf{x}_{i f}\right)
$$

This distance metric has the merit that it allows knowledge to be brought to bear on the assessment of similarity. The set of features $F$ can be chosen to reflect the important features in the domain and knowledge can also be encoded in the design of the $\delta$ function. A basic version 
for continuous and discrete attributes would be:

$$
\delta\left(\mathbf{q}_{f}, \mathbf{x}_{i f}\right)= \begin{cases}0 & f \text { discrete \& } \mathbf{q}_{f}=\mathbf{x}_{i f} \\ 1 & f \text { discrete \& } \mathbf{q}_{f} \neq \mathbf{x}_{i f} \\ \left|\mathbf{q}_{f}-\mathbf{x}_{i f}\right| & f \text { continuous }\end{cases}
$$

The $k$ nearest neighbours are selected based on this distance metric. Then there are a variety of ways in which the $k$ nearest neighbours can be used to determine the class of $\mathbf{q}$. The most straightforward approach is to assign the majority class among the nearest neighbours to the query.

It will often make sense to assign more weight to the nearer neighbours in deciding the class of the query. A fairly general technique to achieve this is distance weighted voting where the neighbours get to vote on the class of the query case with votes weighted by the inverse of their distance to the query as follows:

$$
\operatorname{Vote}\left(y_{j}\right)=\sum_{c=1}^{k} \frac{1}{d\left(\mathbf{q}, \mathbf{x}_{c}\right)^{n}} 1\left(y_{j}, y_{c}\right)
$$

Thus the vote assigned to class $y_{j}$ by neighbour $\mathbf{x}_{c}$ is 1 divided by the distance to that neighbour, i.e. $1\left(y_{j}, y_{c}\right)$ returns 1 if the class labels match and 0 otherwise. In equation $3 n$ would normally be 1 but values greater than 1 can be used to further reduce the influence of more distant neighbours.

\subsection{Similarity and Distance Metrics}

While the terms similarity metric and distance metric are often used colloquially to refer to any measure of affinity between two objects, the term metric has a formal meaning in mathematics. A metric must conform to the following four criteria (where $d(x, y)$ refers to the distance between two objects $x$ and $y$ ):

1. $d(x, y) \geq 0$; non-negativity

2. $d(x, y)=0$ iff $x=y$; identity

3. $d(x, y)=d(y, x)$; symmetry

4. $d(x, z) \leq d(x, y)+d(y, z)$; triangle inequality

It is possible to build a $k$-NN classifier that incorporates an affinity measure that is not a proper metric, however there are some performance optimisations to the basic $k$-NN algorithm that require the use of a proper metric $[19,20]$. In brief, these techniques can identify the nearest neighbour of an object without comparing that object to every other object but the affinity measure must be a metric, in particular it must satisfy the triangle inequality.

The basic distance metric described in equations 1 and 2 is a special case of the Minkowski Distance metric - in fact it is the 1-norm $\left(L_{1}\right)$ Minkowski distance. The general formula for the Minkowski distance is

$$
M D_{p}\left(\mathbf{q}, \mathbf{x}_{i}\right)=\left(\sum_{f \in F}\left|\mathbf{q}_{f}-\mathbf{x}_{i f}\right|^{p}\right)^{\frac{1}{p}}
$$

The $L_{1}$ Minkowski distance is also known as the Manhattan distance and the $L_{2}$ distance is the Euclidean distance. It is unusual but not unheard of to use $p$ values greater than 2. Larger 
values of $p$ have the effect of giving greater weight to the attributes on which the objects differ most. To illustrate this we can consider three points in $2 \mathrm{D}$ space; $A=(1,1), B=(5,1)$ and $C=(4,4)$. Since $A$ and $B$ differ on one attribute only the $M D_{p}(A, B)$ is 4 for all $p$, whereas $M D_{p}(A, C)$ is $6,4.24$ and 3.78 for $p$ values of 1,2 and 3 respectively. So $C$ becomes the nearer neighbour to $A$ for $p$ values of 3 and greater.

The other important Minkowski distance is the $L_{\infty}$ or Chebyshev distance.

$$
M D_{\infty}\left(\mathbf{q}, \mathbf{x}_{i}\right)=\max _{f \in F}\left|\mathbf{q}_{f}-\mathbf{x}_{i f}\right|
$$

This is simply the distance in the dimension in which the two examples are most different; it is sometimes referred to as the chessboard distance as it is the number of moves it takes a chess king to reach any square on the board.

\subsection{Kullback-Leibler Divergence and the $\chi^{2}$ Statistic}

The Minkowski distance defined in (4) is a very general metric that can be used in a $k$-NN classifier for any data that is represented as a feature vector. When working with image data a convenient representation for the purpose of calculating distances is a colour histogram. An image can be considered as a grey-scale histogram $H$ of $N$ levels or bins where $h_{i}$ is the number of pixels that fall into the interval represented by bin $i$ (this vector $h$ is the feature vector). The Minkowski distance formula (4) can be used to compare two images described as histograms. $L_{1}$, $L_{2}$ and less often $L_{\infty}$ norms are used.

Other popular measures for comparing histograms are the Kullback-Leibler divergence (5) [21] and the $\chi^{2}$ statistic (6) [22].

$$
\begin{gathered}
d_{K L}(H, K)=\sum_{i=1}^{N} h_{i} \log \left(\frac{h_{i}}{k_{i}}\right) \\
d_{\chi^{2}}(H, K)=\sum_{i=1}^{N} \frac{h_{i}-m_{i}}{h_{i}}
\end{gathered}
$$

where $H$ and $K$ are two histograms, $h$ and $k$ are the corresponding vectors of bin values and $m_{i}=\frac{h_{i}+k_{i}}{2}$.

While these measures have sound theoretical support in information theory and in statistics they have some significant drawbacks. The first drawback is that they are not metrics in that they do not satisfy the symmetry requirement. However, this problem can easily be overcome by defining a modified distance between $x$ and $y$ that is in some way an average of $d(x, y)$ and $d(y, x)$ - see [22] for the Jeffrey divergence which is a symmetric version of the Kullback-Leibler divergence.

A more significant drawback is that these measures are prone to errors due to bin boundaries. The distance between an image and a slightly darker version of itself can be great if pixels fall into an adjacent bin as there is no consideration of adjacency of bins in these measures.

\subsection{Symbolic Attributes in Taxonomies}

In section 2.2 we mentioned that one way in which structure can be incorporated in the case representation is by organizing feature values into a taxonomy of is-a relationships [16]. In this situation the structure of the taxonomy contains information about the similarity between two feature values. 
Wu and Palmer [23] proposed the following measure to quantify this:

$$
\delta\left(\mathbf{q}_{f}, \mathbf{x}_{i f}\right)=\frac{2 N}{N\left(\mathbf{q}_{f}\right)+N\left(\mathbf{x}_{i f}\right)+2 N}
$$

where $N\left(\mathbf{q}_{f}\right)$ and $N\left(\mathbf{x}_{i f}\right)$ are the number of edges between the corresponding feature values and their common parent $T\left(\mathbf{q}_{f}, \mathbf{x}_{i f}\right)$ in the hierarchy and $N$ is the number of edges between this common parent node and the root of the taxonomy. This measure is interesting in that, in addition to considering the distances to the common parent node, it also considers the distance from that node to the root of the taxonomy. While this edge counting measure is conceptually simple and can be calculated directly it has the potential drawback that it assigns the same status to all edges. It does not capture the fact that some is-a relationships are closer than others. In section 5 we describe an information-theoretic measure for similarity in taxonomies proposed by Resnik that overcomes this [24].

\subsection{Summary}

These direct mechanisms for similarity assessment represent the dominant strategy in CBR research. They have the advantage of being computationally efficient and are effective in most situations. These direct mechanisms are closely tied to the use of feature-based representations in CBR. This view of representation and similarity has had a significant impact on CBR research and has wide ranging implications. It formulates the problem space as a vector space, it emphasises the dimensionality of the data as an issue and it abstracts the similarity mechanism from the raw data.

\section{Transformation-Based Measures}

An alternative perspective on assessing the distance or similarity between objects is the effort required to transform one into the other. This is the principle underlying the notion of edit distance which has quite a long history in Computer Science [25]. Edit distance has already been used in CBR research as an affinity measure $[4,5]$, indeed the whole CBR field of adaptation guided retrieval is based on a view of similarity as 'transformation effort' [26].

\subsection{Edit Distance (Levenshtein Distance)}

The edit distance (ED) is the most basic of these transformation-based measures. It counts the number of insertions, deletions and substitutions required to transform one string to another the ED from cat to rat is 1 and the ED from cats to cat is 1 . Edit distances can be calculated efficiently using dynamic programming and the algorithm is $O\left(n^{2}\right)$ in time and space where $n$ is the string length. In terms of the subject matter of this paper, edit distance has the added importance that it can be augmented with specific knowledge about the data to produce a very knowledge-based (dis)similarity measure - an example of this is presented in the next section.

\subsection{Alignment Measures for Biological Sequences}

The problem of assessing similarity for biological sequences has been receiving attention for many years. There are a variety of sequence alignment problems in biology, e.g. in comparing DNA, RNA or protein sequences. The protein sequences GAATCCG and GATTGC might be aligned as 


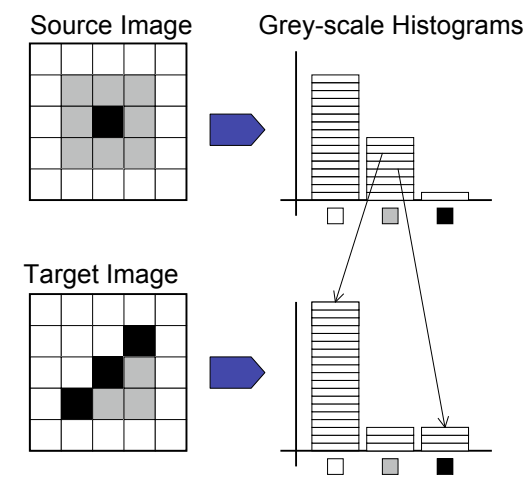

Figure 3: An example of the EMD effort required to transform one image to another with images represented as histograms.

follows $[27]$ :

\section{G-AATCCG-}

GAT-T-G-C

This alignment is scored as the the sum of the contributions of the alignment scores $(\mathrm{G} \leftrightarrow \mathrm{G}$, $\mathrm{A} \leftrightarrow \mathrm{T}, \mathrm{T} \leftrightarrow \mathrm{T}$, etc.) minus penalty terms for the gaps. The alignment scores (e.g. $\mathrm{A} \leftrightarrow \mathrm{T})$ are read from a substitution matrix. For any pair of strings there will be a number of possible alignments and associated scores. The actual alignment score is the one associated with the alignment with the highest score - the Smith-Waterman algorithm determines this using dynamic programming [27]. This is a transformation score that is specialised for the problem at hand with similarity knowledge encoded in this substitution matrix and in the manner the gap penalty is calculated. Alignment scores such as this embody a notion of similarity that is in tune with the way biologists view the data.

\subsection{Earth Mover Distance}

The Earth Mover Distance (EMD) is a transformation-based distance for image data. It overcomes many of the problems that arise from the arbitrariness of binning when using histograms (see section 3.2). As the name implies, the distance is based on an assessment of the amount of effort required to convert one image to another based on the analogy of transporting mass from one distribution to another (see Figure 3).

In their analysis of the EMD Rubner et al. [22] argue that a measure based on the notion of a signature is better than one based on a histogram. A signature $\left\{\mathbf{s}_{j}=\mathbf{m}_{j}, w_{\mathbf{m}_{j}}\right\}$ is a set of $j$ clusters where $\mathbf{m}_{j}$ is a vector describing the mode of cluster $j$ and $w_{\mathbf{m}_{j}}$ is the fraction of pixels falling into that cluster. Thus a signature is a generalisation of the notion of a histogram where boundaries and the number of partitions are not set in advance; instead $j$ should be 'appropriate' to the complexity of the image [22].

For two images described by signatures $S=\left\{\mathbf{m}_{j}, w_{\mathbf{m}_{j}}\right\}_{j=1}^{n}$ and $Q=\left\{\mathbf{p}_{k}, w_{\mathbf{p}_{k}}\right\}_{k=1}^{r}$ we are interested in the work required to transfer from one to the other for a given flow pattern $\mathbf{F}$ :

$$
W O R K(S, Q, \mathbf{F})=\sum_{j=1}^{n} \sum_{k=1}^{r} d_{j k} f_{j k}
$$




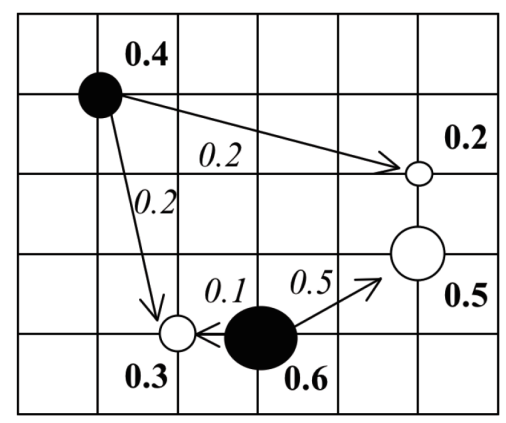

Figure 4: An example of the EMD effort required to transform one image to another with images represented as signatures: the source image is represented by two clusters (black circles) and the target image by three clusters (white circles).

where $d_{j k}$ is the distance between clusters $\mathbf{m}_{j}$ and $\mathbf{p}_{k}$ and $f_{j k}$ is the flow between $\mathbf{m}_{j}$ and $\mathbf{p}_{k}$ that minimises overall cost. An example of this in a 2D colour space is shown in Figure 4. Once the transportation problem of identifying the flow that minimises effort is solved (using dynamic programming) the EMD is defined to be:

$$
\operatorname{EMD}(S, Q)=\frac{\sum_{j=1}^{n} \sum_{k=1}^{r} d_{j k} f_{j k}}{\sum_{j=1}^{n} \sum_{k=1}^{r} f_{j k}}
$$

Efficient algorithms for the EMD are described in [22] however this measure is expensive to compute with cost increasing more than linearly with the number of clusters. Nevertheless it is an effective measure for capturing similarity between images.

\subsection{Similarity for Networks and Graphs}

When a case representation has some structure an effective similarity measure will need to take account of that structure in assessing similarity. This has long been a research topic in CBR (e.g. [1]); indeed it predates CBR as it has been an active research area in analogy and cognitive science since the 1980s $[28,2,14]$.

Perhaps the most famous measure for similarity from research on analogy is the structure mapping engine (SME) $[28,2]$. One of the classic SME examples is shown in Figure 5 [2]. This illustrates the analogy between water flow from a beaker to a vial connected by a tube and heat flow between a cup of coffee and an ice cube connected by a silver bar. As the name suggests, the key step in SME is to identify the appropriate mapping between the two domains. There are two parts to this, the identification of corresponding entities and predicates in the two domains and the construction of a maximal mapping between the structures in the two domains. It is well known now that this problem is NP hard [29] so practical solutions will need to constrain the search in some way or use a greedy search strategy.

From this early work on structural similarity in cognitive science the research has established a more formal computational basis in the wider context of graph (or sub-graph) isomorphism [1, $9,10,16]$. The graph theoretic basis for structure mapping as a similarity measure is laid out very well by Bunke and Messmer [1] and by Bergmann [16]. Bergmann identifies two distinct strategies for assessing similarity between graphs: these are graph matching measures and mechanisms based on edit distance. The most fundamental graph matching problem is (complete) graph 


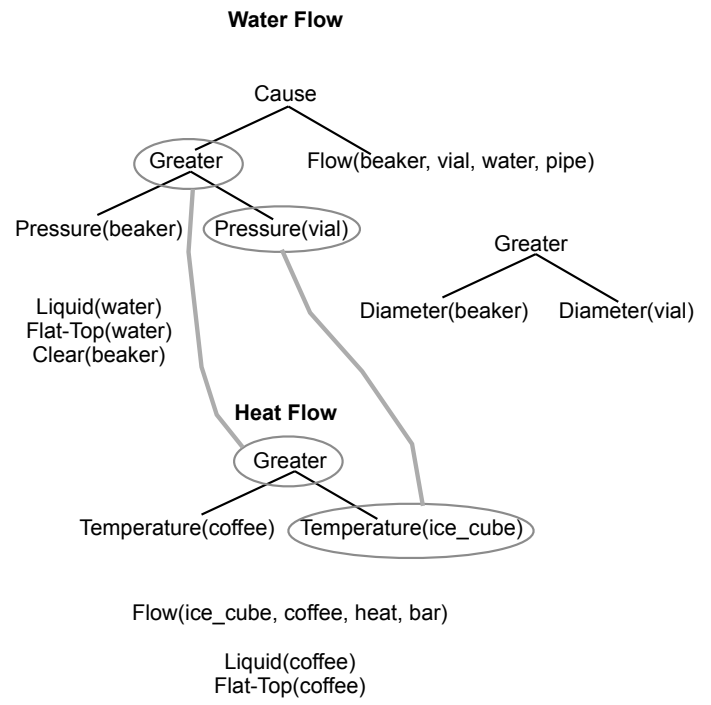

Figure 5: This diagram shows a classic SME example from [2]. The analogy is between a water flow example and a heat flow example - water flows from a beaker to a vial and heat flows from a coffee cup to an ice-cube. Some entities and predicates that map between the two domains are highlighted, e.g. the water pressure in the vial is analogous to the temperature in the ice-cube.

isomorphism, however this is less relevant in CBR because exact matches are unlikely to occur. Instead sub-graph isomorphism and the problem of identifying the largest common subgraph are the relevant graph matching measures. It is interesting that the most recent work on structural similarity in CBR [9] employs graph edit-distance which belongs to the second set of strategies described by Bergmann.

The work by Minor et al [9] is based on the original graph edit-distance framework proposed by Bunke and Messmer [1]. They propose that each case is represented as a directed graph $g=(N, E, \alpha, \beta)$ where;

$-N$ is a finite set of nodes,

- $E \subseteq N \times N$ is the finite set of edges,

- $\alpha: N \mapsto L_{N}$ is the node labeling function,

$-\beta: E \mapsto L_{E}$ is the edge labeling function.

In this framework the distance between two graphs $g$ and $g^{\prime}$ is the shortest sequence of edit operations that converts $g$ to $g^{\prime}$. A sequence of edit operations can be denoted $s=\left(e_{1}, e_{2}, \ldots, e_{n}\right)$ and the cost of the sequence is $c(s)=\sum_{i=1}^{n} c\left(e_{i}\right)$ where $c\left(e_{i}\right)$ is the cost of edit operation $e_{i}$. The distance measure based on this is:

$$
d\left(g, g^{\prime}\right)=\min \left\{\sum_{i=1}^{n} c\left(e_{i}\right)\right\}
$$

where $\left(e_{1}, \ldots, e_{n}\right)$ transforms $q$ to $q^{\prime}$ 

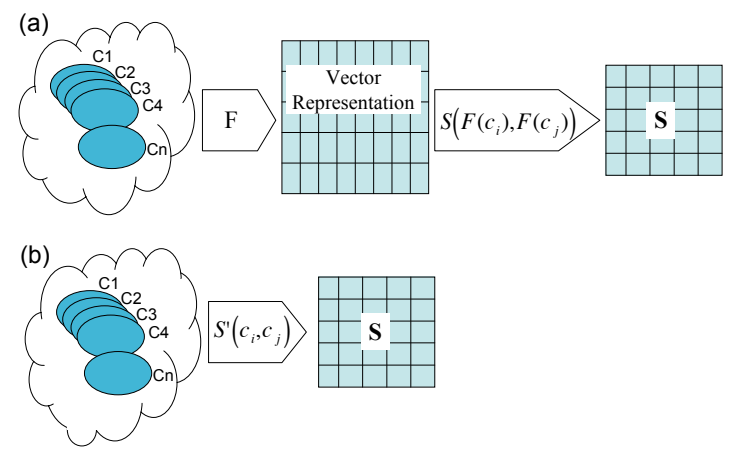

Figure 6: A comparison of Feature-based (a) and Featureless (b) similarity assessment - in (a) F is the Feature Extraction process, $S\left(F\left(c_{i}\right), F\left(c_{j}\right)\right)$ scores similarity based on features extracted from the cases and in (b) $S^{\prime}\left(c_{i}, c_{j}\right)$ operates more directly on the raw case data.

The edit operations are; deletion, insertion or substitution of edges or nodes $(3 \times 2=6$ types of operation in all). The sub-graph isomorphism problem remains NP-complete when cast in this edit-distance framework. Bunke and Messmer show that for practical problems the computational complexity can be relieved somewhat by maintaining a library of commonly occurring subgraphs which they call models. Minor et al. show that this graph edit distance approach to similarity for structured cases is effective for the real-world problem of workflow management.

\section{Information Theoretic Measures}

It should not be surprising that information theory offers a variety of techniques for assessing the similarity of two objects. Perhaps the most dramatic of these is compression-based similarity. A compression-based approach to similarity has the advantage that it works directly on the raw case representation (see Figure 6(b)) thus it avoids the feature extraction process that produces the feature vector representation where information might be lost (Figure 6(a)). Delany and Bridge [3] refer to this as a feature-free approach to case representation.

\subsection{Compression-Based Similarity for Text}

In recent years the idea of basing a similarity metric on compression has received a lot of attention $[30,31]$. Indeed Li et al. [30] refer to this as The similarity metric. The basic idea is quite straightforward; if two documents are very similar then the compressed size of the two documents concatenated together will not be much greater than the compressed size of a single document. This will not be true for two documents that are very different. Slightly more formally, the difference between two documents $A$ and $B$ is related to the compressed size of document $B$ when compressed using the codebook produced when compressing document $A$.

The theoretical basis of this metric is in the field of Kolmogorov complexity, specifically in conditional Kolmogorov complexity [30]. A definition of similarity based on Kolmogorov complexity is:

$$
d_{K v}(x, y)=\frac{K v(x \mid y)+K v(y \mid x)}{K v(x y)}
$$


where $K v(x)$ is the length of the shortest program that computes $x, K v(x \mid y)$ is the length of the shortest program that computes $x$ when $y$ is given as an auxiliary input to the program and $K v(x y)$ is the length of the shortest program that outputs $y$ concatenated to $x$. While this is an abstract idea it can be approximated using compression

$$
d_{C}(x, y)=\frac{C(x \mid y)+C(y \mid x)}{C(x y)}
$$

$C(x)$ is the size of data $x$ after compression, and $C(x \mid y)$ is the size of $x$ after compressing it with the compression model built for $y$. If we assume that $K v(x \mid y) \approx K v(x y)-K v(y)$ then we can define a practical compression distance

$$
d_{N C}(x, y)=\frac{C(x y)-\min (C(x), C(y))}{\max (C(x), C(y))}
$$

It is important that $C($.$) should be an appropriate compression metric for the type of data.$ Delany and Bridge [3] show that compression using Lempel-Ziv (GZip) is effective for text. They show that this compression based metric is more accurate in $k$-NN classification than distancebased metrics using a bag-of-words representation of the text (i.e. a feature vector).

\subsection{Information-Based Similarity for Biological Sequences}

An interesting characteristic of gene sequence data is that the data is typically not compressible using standard (text) compression techniques [32]. Information theory tells us that biological sequences should be compressible because they encode information, i.e. they are not random. However, the regularity in biological sequences is more subtle than in text, thus specialised algorithms are required to compress them. Li. et al. [33] have shown that a compression based similarity metric can be very effective for phylogenitic studies provided that a compression algorithm specialised for the data is used - they use the GenCompress algorithm developed by Chen et al. [32] which is based on approximate matching. This research is of general importance because it illustrates a novel strategy to bring domain knowledge to bear in assessing similarity.

It is worth mentioning that, even though we have included this in the Information-theoretic category, it has some of the characteristics of a Transformation-based metric.

\subsection{Similarity in a Taxonomy}

An edge-counting method for assessing the similarity between two feature values in a taxonomy in shown in equation 7. As stated already, this has the drawback that it does not capture the fact that some is-a relationships are closer than others. Resnik has proposed an informationtheoretic measure that overcomes this to a large extent [24]. It is based on the idea that the information content of a concept in a taxonomy can be quantified as the negative log liklihood $-\log (\mathrm{p}(c))$ where $\mathrm{p}(c)$ is the probability of observing the concept $c$. If the taxonomy has a single root then the information content of the root node is 0 . In this framework the similarity between two features is the information content of the common parent node with the highest information content:

$$
\delta\left(\mathbf{q}_{f}, \mathbf{x}_{i f}\right)=\max _{c \in\left\{S\left(\mathbf{q}_{f}, \mathbf{x}_{i f}\right)\right\}}-\log (\mathrm{p}(c))
$$

here $S\left(\mathbf{q}_{f}, \mathbf{x}_{i f}\right)$ represents the common parent concepts of the two feature values under consideration. Other than in situations of multiple inheritance this concept $c$ will be $T\left(\mathbf{q}_{f}, \mathbf{x}_{i f}\right)$ the 
first common parent. Then the similarity is simply $\mathrm{p}\left(T\left(\mathbf{q}_{f}, \mathbf{x}_{i f}\right)\right)$, the frequency of occurrence of $T\left(\mathbf{q}_{f}, \mathbf{x}_{i f}\right)$ and its descendants in the training data.

This measure for similarity within taxonomies has performed well in evaluations and is worthy of consideration $[24,34]$.

\section{Emergent Measures}

The great increase in computing power available in recent years has resulted in a sea change in ML research objectives. Speeding up algorithms is less important now, instead the challenge is to find ways to usefully exploit the power available [35]. Techniques that represent this new direction in ML research are; Random Forests [36], Ensemble Clustering [37], and Stability-based cluster validation [38]. These might be viewed as analytic processes that are allowed to simmer for an extended period to produce a characterisation of the data. This characterisation of the data may present a significant opportunity for knowledge discovery. Of interest here are novel similarity scores that emerge from this characterisation. The three techniques we consider here are Random Forests and Cluster Kernels and Web-Based Kernels.

In analogy with the way feature-based representations can be internal or external as described in section 2.1.1, these emergent mechanisms can be categorised as internal or external. Random Forests and Cluster Kernels are internal in that they uncover new relationships from an analysis within the data set whereas Web-based Kernels bring new knowledge to bear from outside the data set by mining the web.

\subsection{Random Forests}

A Random Forest is an ensemble of decision trees [36]. The general strategy for generating a Random Forest is as follows:

1. For each ensemble member the training set $D$ is sub-sampled with replacement to produce a training set of size $|D|$. (The remaining cases are referred to as the out-of-bag (OOB) cases for that ensemble member).

2. Where $F$ is the set of features that describes the data, $m<<|F|$ is selected as the number of features to be used in the feature selection process. At each stage (i.e. node) in the building of a tree, $m$ features are selected at random to be the candidates for splitting at that node.

In order to ensure diversity among the component trees, no pruning is employed as would be normal in building decision trees. It is normal when building a Random Forest to generate many more ensemble members than would be used in other ensemble techniques - 100 or even 1000 trees might be built. The effort expended on building these trees has the added benefit of providing an analysis of the data.

In particular a novel similarity measure emerges from all of these trees. The idea is to track the frequency with which cases (both training and OOB) are located at the same leaf node. Every leaf node in every tree is examined and a $|D| \times|D|$ matrix is maintained where cell $(i, j)$ is incremented each time cases $i$ and $j$ share the same leaf node. If the matrix entries are divided by the number of trees we have a proximity measure that is in tune with the classification algorithm (the Random Forest). In [39] we have shown that this similarity metric is more effective than a conventional feature-based similarity metric on a wide range of classification tasks. 


\subsection{Cluster Kernels}

Cluster Kernels are relevant in the context of semi-supervised learning where only some of the available data is labelled [40]. Cluster Kernels allow the unlabelled data to influence similarity. This is driven by the cluster assumption that class labels do not change in regions of high density - instead there should be some correspondence between cluster boundaries and the unknown class boundaries. Thus the Cluster Kernel is a composition of a standard kernel built from the labelled data and a kernel derived from clustering all the data. This is a general principle and one embodiment of this idea for protein sequence classification is [41]:

$$
K\left(x_{i}, x_{j}\right)=K_{\text {orig }}\left(x_{i}, x_{j}\right) \cdot K_{\text {bag }}\left(x_{i}, x_{j}\right)
$$

where $K_{\text {orig }}()$ is a basic neighbourhood kernel and $K_{b a g}()$ is a kernel derived from repeated clustering of all the data. $K_{b a g}\left(x_{i}, x_{j}\right)$ is essentially a count of the number of times $x_{i}$ and $x_{j}$ turned up in the same cluster (this is in the same spirit as the strategy used in Random Forests). Thus we have a mechanism that adjusts the similarity metric using measures drawn from repeated clustering of the labelled and unlabelled data. Evaluations of the use of Cluster Kernels in semi-supervised learning shows promising results [41] [40].

\subsection{Web-Based Kernel}

One way to discover that two phrases are related would be to find that they are connected by documents returned in web search. This is the approach taken by Sahami and Heilman [42] in their Web-based Kernel for text snippet similarity. The basic idea is that the sets of documents returned when two text snippets are submitted as web queries will show similarities if the text snippets are related - even if the snippets have no terms in common.

An example of a Web-Based Kernel in operation is shown in Figure 7. The two text snippets to be compared are $x$ and $y$ and the vectors corresponding to the return sets from presenting these to a web search engine are $R(x)$ and $R(y)$. The return sets are restricted to the first $n$ documents and each document is represented by $m$ terms selected by the standard term-frequency inversedocument frequency (TFIDF) criterion. The texts snippets can now be represented by $C(x)$ and $C(y)$ the centroids of $R(x)$ and $R(y)$. The Web-Based Kernel is simply the dot-product of $C(x)$ and $C(y)$. Sahami and Heilman [42] show that similarity based on this strategy is very effective for the task of related query suggestion.

\section{Implications for CBR Research}

The new perspectives on similarity outlined in this paper do not represent a paradigm shift in CBR research since direct assessment of similarity based on a feature value representation will still be the dominant strategy. Instead what we have is a selection of new methodologies for similarity in CBR that may be used as alternatives to the direct strategy in some circumstances. These new methodologies have two important ramifications for CBR:

- In some circumstances (e.g. information-theoretic measures) the role of the vocabulary knowledge container [43] is de-emphasised and the role of the similarity knowledge container is increased.

- Because these new methodologies are typically computationally intensive the importance of strategies for speeding up case-retrieval is increased.

These issues will be discussed in the following sections. 


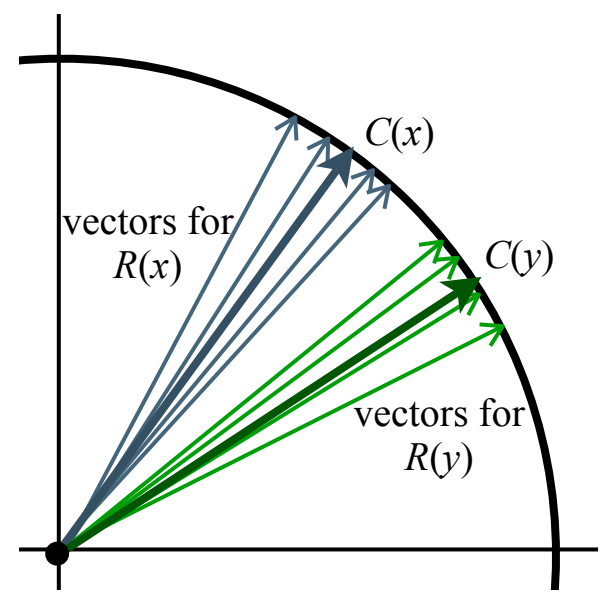

Figure 7: This figure shows the principle underlying the Web-based Kernel. $R(x)$ and $R(y)$ are the vectors corresponding to the return sets for $x$ and $y . C(x)$ and $C(y)$ are the centroid vectors for these vectors.

\subsection{De-emphasising the Vocabulary Knowledge Container}

A popular perspective on the organisation of knowledge in CBR is Richter's knowledge containers model [43] - there are four containers:

1. The case description language (vocabulary)

2. The similarity measure

3. The solution transformation knowledge (adaptation knowledge)

4. The cases themselves

An important aspect of the knowledge containers' view of CBR is the way it highlights the high-level design decisions in the development of a CBR system. Different design choices have the effect of moving knowledge from one container to another. Clearly the information-theoretic strategies described in section 5 move knowledge from the vocabulary knowledge container into the similarity measure.

In our early work on case-based spam filtering we worked with a vector-space representation of messages [44]. This approach meant that careful consideration had to be given to feature extraction and feature selection strategies. Feature selection in the context of concept drift is a particular problem. The solution we adopted required a periodic feature re-selection process. This is all much more straightforward when compression-based similarity is used [3] as there is no feature extraction and selection.

This is also true for transformation-based measures such as edit distance and string alignment kernels. However, the EMD does have a feature extraction stage where the signature is created using clustering - the granularity of this signature is key to the effectiveness of the algorithm. The important point is that the use of novel similarity measures can greatly simplify the design of other aspects of the system. 


\subsection{Computational Complexity}

Computationally expensive metrics such as the EMD and compression based (dis)similarity metrics focus attention on the computational issues associated with case-based classifiers. Basic case-based classifiers that use a simple Minkowski distance will have a time behaviour that is $O(|D||F|)$ where $D$ is the training set and $F$ is the set of features that describe the data, i.e. the distance metric is linear in the number of features and the comparison process increases linearly with the amount of data. The computational complexity of the EMD and compression metrics is more difficult to characterise but a case-based classifier that incorporates an EMD metric is likely to be $O\left(|D| n^{3} \log n\right)$ where $n$ is the number of clusters [22]. The computational cost of compression-based similarity depends on the compression algorithm - for text GZip is roughly linear while PPM is $O\left(n^{2}\right)$ [45]. Even the linear time GZip is much slower than a feature-based measure. Delany and Bridge show that compression-based similarity using GZip can be 200 times slower than the feature-based alternative [3]. There has been considerable research on alternatives to the exhaustive search strategy that is used in the standard $k$-NN algorithm. Here is a summary of four of the strategies for speeding up nearest-neighbour retrieval:

- Case-Retrieval Nets: Case-Retrieval Nets (CRNs) are perhaps the most popular technique for speeding up the retrieval process. The cases are pre-processed to form a network structure that is used at retrieval time. The retrieval process is done by spreading activation in this network structure. CRNs can be configured to return exactly the same cases as $k$-NN $[46,47]$. However, CRNs depend on a feature-based case representation.

- Footprint-Based Retrieval: As with all strategies for speeding up nearest-neighbour retrieval, Footprint-Based Retrieval involves a preprocessing stage to organise the training data into a two level hierarchy on which a two stage retrieval process operates. The preprocessing constructs a competence model which identifies 'footprint' cases which are landmark cases in the data. This process is not guaranteed to retrieve the same cases as $k$-NN but the results of the evaluation of speed-up and retrieval quality are nevertheless impressive [48].

- Fish \& Shrink: This technique requires the distance to be a true metric as it exploits the triangle inequality property to produce an organisation of the case-base into candidate neighbours and cases excluded from consideration. Cases that are remote from the query can be bounded out so that they need not be considered in the retrieval process. Fish \& Shrink can be guaranteed to be equivalent to $k$-NN [20].

- Cover Trees for Nearest Neighbor: This technique might be considered the stateof-the-art in nearest-neighbour speed-up. It uses a data-structure called a Cover Tree to organise the cases for efficient retrieval. The use of Cover Trees requires that the distance measure is a true metric, however they have attractive characteristics in terms of space requirements and speed-up performance. The space requirement is $O(n)$ where $n$ is the number of cases; the construction time is $O\left(c^{6} n \log n\right)$ and the retrieval time is $O\left(c^{12} \log n\right)$ where $c$ is a measure of the inherent dimensionality of the data [19].

While CRNs have no role in systems that incorporate featureless similarity, the other three techniques are applicable and will help to make these expensive similarity measures more generally usable. 


\section{Conclusion}

This paper presents an attempt to organise the broad range of strategies for similarity assessment in CBR into a coherent taxonomy. The taxonomy has four main categories; Direct, Transformation-Based, Information-Theoretic and Emergent measures. We have emphasised the centrality of feature-value representation of cases coupled with direct similarity measures in the CBR paradigm. We have argued that in some circumstances benefits can accrue from the use of some of the alternative similarity measures reviewed here. The benefit might be that the overall design of the system is simplified or it may be that the alternative metric simply offers better accuracy because it embodies specific knowledge about the data. This is very much in line with current research on SVMs where it is understood that the effectiveness of the classifier depends very much on the appropriateness of the kernel function for the data (i.e. in encoding domain knowledge in the kernel function).

Thus there are two considerations for CBR research. The first is that a broader perspective on similarity along the lines discussed in this paper may be useful. The second is that the practicality of these computationally expensive similarity measures may depend on clever retrieval techniques such as Cover Trees to make them computationally tractable.

\section{Acknowledgements}

The author is grateful to Derek Bridge, Sarah Jane Delany and Jean Lieber for helpful discussions about this work. This research was supported by Science Foundation Ireland Grant No. 05/IN.1/I2.

\section{References}

[1] Bunke, H., Messmer, B.T.: Similarity measures for structured representations. In Wess, S., Althoff, K.D., Richter, M.M., eds.: EWCBR. Volume 837 of Lecture Notes in Computer Science., Springer (1993) 106-118

[2] Falkenhainer, B., Forbus, K.D., Gentner, D.: The structure-mapping engine. In: AAAI. (1986) 272-277

[3] Delany, S.J., Bridge, D.G.: Catching the drift: Using feature-free case-based reasoning for spam filtering. In Weber, R., Richter, M.M., eds.: ICCBR. Volume 4626 of Lecture Notes in Computer Science., Springer (2007) 314-328

[4] Arcos, J.L., Grachten, M., de Mántaras, R.L.: Extracting performers' behaviors to annotate cases in a cbr system for musical tempo transformations. In Ashley, K.D., Bridge, D.G., eds.: ICCBR. Volume 2689 of Lecture Notes in Computer Science., Springer (2003) 20-34

[5] Costello, E., Wilson, D.C.: A case-based approach to gene finding. In: Proceedings of the Fifth International Conference on Case-Based Reasoning Workshop on CBR in the Health Sciences. (2003) 19-28

[6] Wiratunga, N., Koychev, I., Massie, S.: Feature selection and generalisation for retrieval of textual cases. In Funk, P., González-Calero, P.A., eds.: ECCBR. Volume 3155 of Lecture Notes in Computer Science., Springer (2004) 806-820 
[7] Gabrilovich, E., Markovitch, S.: Overcoming the brittleness bottleneck using Wikipedia: Enhancing text categorization with encyclopedic knowledge. In: Proceedings of the 21st National Conference on Artificial Intelligence, AAAI Press (2006) 1301-1306

[8] Bergmann, R., Stahl, A.: Similarity measures for object-oriented case representations. In Smyth, B., Cunningham, P., eds.: EWCBR. Volume 1488 of Lecture Notes in Computer Science., Springer (1998) 25-36

[9] Minor, M., Tartakovski, A., Bergmann, R.: Representation and structure-based similarity assessment for agile workflows. In Weber, R.O., Richter, M.M., eds.: Case-Based Reasoning Research and Development, 7th International Conference on Case-Based Reasoning, ICCBR 2007, Belfast, Northern Ireland, UK, August 2007, Proceedings. LNCS 4626, Springer (2007) $224-238$

[10] Plaza, E.: Cases as terms: A feature term approach to the structured representation of cases. In Veloso, M.M., Aamodt, A., eds.: ICCBR. Volume 1010 of Lecture Notes in Computer Science., Springer (1995) 265-276

[11] Sanders, K.E., Kettler, B.P., Hendler, J.A.: The case for graph-structured representations. In Leake, D.B., Plaza, E., eds.: ICCBR. Volume 1266 of Lecture Notes in Computer Science., Springer (1997) 245-254

[12] Smyth, B., Cunningham, P.: Déjà vu: A hierarchical case-based reasoning system for software design. In: ECAI. (1992) 587-589

[13] Smyth, B., Keane, M.T., Cunningham, P.: Hierarchical case-based reasoning integrating case-based and decompositional problem-solving techniques for plant-control software design. IEEE Trans. Knowl. Data Eng. 13 (2001) 793-812

[14] Keane, M.T., Brayshaw, M.: The incremental analogy machine: A computational model of analogy. In: EWSL. (1988) 53-62

[15] Veloso, M.M., Carbonell, J.G.: Case-based reasoning in ProdiGy. In Michalski, R.S., Teccuci, G., eds.: Machine Learning: A Multistrategy Approach, Volume IV. Morgan Kaufmann (1994) 523-548

[16] Bergmann, R.: Experience Management: Foundations, Development Methodology, and Internet-Based Applications. Volume 2432 of Lecture Notes in Computer Science. Springer (2002)

[17] Lenz, M., Ashley, K.: Proceedings of the AAAI98 Workshop On Textural Case-Based Reasoning. AAAI press (1998)

[18] Shimazu, H.: A textual case-based reasoning system using xml on the world-wide web. In Smyth, B., Cunningham, P., eds.: Advances in Case-Based Reasoning: Proceedings of the Fourth European Workshop on Case-Based Reasoning. Volume 1488 of Lecture Notes in Computer Science., Springer (1998) 274-285

[19] Beygelzimer, A., Kakade, S., Langford, J.: Cover trees for nearest neighbor. In: Proceedings of 23rd International Conference on Machine Learning (ICML 2006). (2006)

[20] Schaaf, J.: Fish and Shrink. A Next Step Towards Efficient Case Retrieval in Large-Scale Case Bases. In Smith, I., Faltings, B., eds.: European Conference on Case-Based Reasoning (EWCBR'96, Springer (1996) 362-376 
[21] Kullback, S., Leibler, R.A.: On information and sufficiency. Annals of Mathematical Statistics 22 (1951) 79-86

[22] Rubner, Y., Tomasi, C., Guibas, L.J.: The earth mover's distance as a metric for image retrieval. International Journal of Computer Vision 40 (2000) 99-121

[23] Wu, Z., Palmer, M.S.: Verb semantics and lexical selection. In: ACL. (1994) 133-138

[24] Resnik, P.: Using information content to evaluate semantic similarity in a taxonomy. In: IJCAI. (1995) 448-453

[25] Levenshtein, V.: Binary codes capable of correcting deletions, insertions, and reversals. Problems in Information Transmission 1 (1965) 8-17

[26] Smyth, B., Keane, M.T.: Adaptation-guided retrieval: Questioning the similarity assumption in reasoning. Artif. Intell. 102 (1998) 249-293

[27] Vert, J.P., Saigo, H., Akutsu, T.: Local alignment kernels for biological sequences. In Schölkopf, B., Tsuda, K., Vert, J.P., eds.: Kernel Methods in Computational Biology. MIT Press (2004)

[28] Gentner, D.: Structure-mapping: A theoretical framework for analogy. Cognitive Science 7 (1983) $155-170$

[29] Veale, T., Keane, M.T.: The competence of sub-optimal theories of structure mapping on hard analogies. In: IJCAI (1). (1997) 232-237

[30] Li, M., Chen, X., Li, X., Ma, B., Vitányi, P.M.B.: The similarity metric. IEEE Transactions on Information Theory 50 (2004) 3250-3264

[31] Keogh, E.J., Lonardi, S., Ratanamahatana, C.: Towards parameter-free data mining. In Kim, W., Kohavi, R., Gehrke, J., DuMouchel, W., eds.: KDD, ACM (2004) 206-215

[32] Chen, X., Kwong, S., Li, M.: A compression algorithm for DNA sequences and its applications in genome comparison. Proceedings of RECOMB 107 (2000)

[33] Li, M., Badger, J.H., Chen, X., Kwong, S., Kearney, P.E., Zhang, H.: An information-based sequence distance and its application to whole mitochondrial genome phylogeny. Bioinformatics 17 (2001) 149-154

[34] Bolshakova, N., Azuaje, F., Cunningham, P.: Incorporating biological domain knowledge into cluster validity assessment. In Rothlauf, F., Branke, J., Cagnoni, S., Costa, E., Cotta, C., Drechsler, R., Lutton, E., Machado, P., Moore, J.H., Romero, J., Smith, G.D., Squillero, G., Takagi, H., eds.: EvoWorkshops. Volume 3907 of Lecture Notes in Computer Science., Springer (2006) 13-22

[35] Esmeir, S., Markovitch, S.: Anytime induction of decision trees: An iterative improvement approach. In: Proceedings of the 21st National Conference on Artificial Intelligence, AAAI Press (2006) 348-355

[36] Breiman, L.: Random forests. Machine Learning 45 (2001) 5-32

[37] Greene, D., Tsymbal, A., Bolshakova, N., Cunningham, P.: Ensemble clustering in medical diagnostics. In: CBMS, IEEE Computer Society (2004) 576-581 
[38] Lange, T., Roth, V., Braun, M.L., Buhmann, J.M.: Stability-based validation of clustering solutions. Neural Computation 16 (2004) 1299-1323

[39] Tsymbal, A., Pechenizkiy, M., Cunningham, P.: Dynamic integration with random forests. In Fürnkranz, J., Scheffer, T., Spiliopoulou, M., eds.: ECML. Volume 4212 of Lecture Notes in Computer Science., Springer (2006) 801-808

[40] Chapelle, O., Weston, J., Schölkopf, B.: Cluster kernels for semi-supervised learning. In Becker, S., Thrun, S., Obermayer, K., eds.: NIPS, MIT Press (2002) 585-592

[41] Weston, J., Leslie, C.S., Ie, E., Zhou, D., Elisseeff, A., Noble, W.S.: Semi-supervised protein classification using cluster kernels. Bioinformatics 21 (2005) 3241-3247

[42] Sahami, M., Heilman, T.: A web-based kernel function for measuring the similarity of short text snippets. In: Proceedings of the 15th international conference on World Wide Web, ACM Press New York, NY, USA (2006) 377-386

[43] Richter, M.M.: Introduction. In Lenz, M., Bartsch-Spörl, B., Burkhard, H.D., Wess, S., eds.: Case-Based Reasoning Technology. Volume 1400 of Lecture Notes in Computer Science., Springer (1998) 1-16

[44] Delany, S., Cunningham, P., Smyth, B.: ECUE: A spam filter that uses machine learning to track concept drift. In Brewka, G., S, C., Perini, A., Traverso, P., eds.: 17th European Conference on Artificial Intelligence (ECAI-06), IOS Press (2006) 627-631

[45] Bell, T.C., Witten, I.H., Cleary, J.G.: Text Compression. Prentice Hall (1990)

[46] Lenz, M., H.-D.Burkhard, Brückner, S.: Applying case retrieval nets to diagnostic tasks in technical domains. In Smith, I.F.C., Faltings, B., eds.: EWCBR. Volume 1168 of Lecture Notes in Computer Science., Springer (1996) 219-233

[47] Lenz, M., Burkhard, H.D.: Case retrieval nets: Basic ideas and extensions. In: KI Kunstliche Intelligenz. (1996) 227-239

[48] Smyth, B., McKenna, E.: Footprint-based retrieval. In Althoff, K.D., Bergmann, R., Branting, K., eds.: ICCBR. Volume 1650 of Lecture Notes in Computer Science., Springer (1999) 343-357 